\title{
Scattering cross section of metal catalyst atoms in silicon nanowires
}

\author{
Markussen, Troels; Rurali, R.; Cartoixa, X.; Jauho, Antti-Pekka; Brandbyge, Mads
}

Published in:

Physical Review B Condensed Matter

Link to article, DOI:

10.1103/PhysRevB.81.125307

Publication date:

2010

Document Version

Publisher's PDF, also known as Version of record

Link back to DTU Orbit

Citation (APA):

Markussen, T., Rurali, R., Cartoixa, X., Jauho, A-P., \& Brandbyge, M. (2010). Scattering cross section of metal catalyst atoms in silicon nanowires. Physical Review B Condensed Matter, 81(12), 125307.

https://doi.org/10.1103/PhysRevB.81.125307

\section{General rights}

Copyright and moral rights for the publications made accessible in the public portal are retained by the authors and/or other copyright owners and it is a condition of accessing publications that users recognise and abide by the legal requirements associated with these rights.

- Users may download and print one copy of any publication from the public portal for the purpose of private study or research.

- You may not further distribute the material or use it for any profit-making activity or commercial gain

- You may freely distribute the URL identifying the publication in the public portal 


\title{
Scattering cross section of metal catalyst atoms in silicon nanowires
}

\author{
Troels Markussen, ${ }^{1, *}$ Riccardo Rurali, ${ }^{2,3}$ Xavier Cartoixà, ${ }^{3}$ Antti-Pekka Jauho, ${ }^{1,4}$ and Mads Brandbyge ${ }^{1}$ \\ ${ }^{1}$ Department of Micro- and Nanotechnology, Technical University of Denmark, DTU Nanotech, DK-2800 Kgs. Lyngby, Denmark \\ ${ }^{2}$ Institut de Ciència de Materials de Barcelona (ICMAB-CSIC), Campus de Bellaterra, 08193 Bellaterra, Barcelona, Spain \\ ${ }^{3}$ Departament d'Enginyeria Electrònica, Universitat Autònoma de Barcelona, 08193 Bellaterra, Barcelona, Spain \\ ${ }^{4}$ Department of Applied Physics, Aalto University, P. O. Box 11100, FI-00076 AALTO, Finland
}

(Received 15 December 2009; published 8 March 2010)

\begin{abstract}
A common technique to fabricate silicon nanowires is to use metal particles (e.g., $\mathrm{Au}, \mathrm{Ag}, \mathrm{Cu}, \mathrm{Al}$ ) to catalyze the growth reaction. As a consequence, the fabricated nanowires contain small concentrations of these metals as impurities. In this work we investigate the effect of the metallic impurities on the electronic transport properties of silicon nanowires. The computational method is based on $a b$ initio density functional theory together with nonequilibrium Green's functions. From the computed transmission functions we extract a scattering cross section to characterize the scattering strength of the different metal atoms. We find that Au, Ag, and $\mathrm{Cu}$ impurities have very similar scattering cross sections, while $\mathrm{Al}$ differs from the rest. Impurities located in the center of the wires scatter significantly more than impurities close to or at the surface. The results for nanowires are compared with bulk Si scattering calculations and good agreement is found. This agreement shows that the scattering results for the ultrathin nanowires (which are computationally feasible) are not dominated by finite size or surface effects, and indicate that the results can be extended to larger and experimentally more relevant wires.
\end{abstract}

DOI: 10.1103/PhysRevB.81.125307

PACS number(s): 73.63.-b, 72.10.Fk

\section{INTRODUCTION}

Silicon nanowires (SiNWs) have attracted enormous attention in the past decade and they show great promise in a variety of applications. Numerous nanoelectronic devices have been demonstrated, ${ }^{1-5}$ as well as biochemical sensors. ${ }^{6}$ More recently, nanowires have shown promise in energy conversion devices. ${ }^{7,8}$ Several theoretical works have also been concerned with structural, electronic and transport properties of SiNWs, see Ref. 9 for a recent review.

The nanowires are typically synthesized by chemical vapor deposition (CVD) via the vapor-liquid-solid (VLS) growth process. ${ }^{10}$ In the VLS growth the semiconductor material precipitates from a supersaturated liquid alloy containing the semiconductor material within a metallic catalyst particle. The nanowire diameter is controlled by the size of the metal particle. Using monodispersed metal nanoparticles, nanowires with controllable diameters below $10 \mathrm{~nm}$ can be produced. ${ }^{5,11,12} \mathrm{Au}$ is typically used as catalyst material ${ }^{5,10-13}$ but other metals such as $\mathrm{Ag},{ }^{14} \mathrm{Al},{ }^{15} \mathrm{Cu},{ }^{16,17}$ and $\mathrm{Pt}$ (Ref. 18) have also been employed.

During the growth process some of the metal atoms will be incorporated in the nanowire as either substitutional or interstitial impurities. ${ }^{19}$ Recent experiments have revealed that the Au concentration in $\mathrm{Si}$ and InAs nanowires can be larger than the bulk solubility limit. ${ }^{19,20}$ It is known that $\mathrm{Au}$ impurities affect the carrier lifetime in $\mathrm{Si}^{21}$ Also, the impurities will act as scattering centers reducing the conductance of the wires.

In this work we apply $a b$ initio density functional theory (DFT) in combination with nonequilibrium Green's function (NEGF) theory to compute the scattering properties of metal impurities in silicon nanowires (SiNWs). We consider SiNWs oriented in the $\langle 110\rangle$ and $\langle 111\rangle$ directions with diameters $D=12-17 \AA$. We characterize the influence of a single impurity by the transmission function and by a scattering cross section, $\sigma$, which we calculate using two different approaches: in the first approach, we calculate the transmission through an infinite hydrogen passivated SiNW containing a single impurity. From the single-impurity transmission we estimate the mean free path, $l_{e}$, as in Ref. 22, and extract the scattering cross section as $\sigma=\left(n l_{e}\right)^{-1}$, with $n$ being the impurity concentration. Due to computational limitations only ultrathin wires with diameters $D \leqslant 2 \mathrm{~nm}$ can be studied in this way. In the second approach we calculate the scattering cross section of Au impurities in bulk $\mathrm{Si}$. In this approach which is applicable to larger wires, we avoid any surface effects. Both approaches predict that $\mathrm{Au}$ impurities scatter electrons less than holes with cross sections $\sigma_{e} \sim 50 \AA^{2}$ and $\sigma_{h} \sim 200 \AA^{2}$, respectively. The agreement among the wire and bulk calculations indicate that results obtained for even very thin wires can be applicable to larger and experimentally more relevant sized wires. Among the different metal catalysts, we find that the group 11 metals $(\mathrm{Cu}, \mathrm{Ag}$, and $\mathrm{Au})$ behave in a very similar manner, while $\mathrm{Al}$ (group 13) differs substantially, especially at energies close to the band edges.

The rest of the paper is organized as follows: in Sec. II we explain the calculational methods. Results are presented in Sec. III and discussed in Sec. IV. Finally, we present our conclusions in Sec. V.

\section{METHOD}

The atomic and electronic structure of the SiNWs are calculated using local orbital DFT calculations as implemented in the SIESTA package. ${ }^{23}$ Silicon atoms are described by an optimized ${ }^{24}$ single- $\zeta$ polarized basis set ( $s, p$, and $d$ orbitals), while hydrogen is described by a single $s$ orbital. The metal atoms are described by a double- $\zeta$ polarized basis set. We use norm-conserving pseudopotentials and the generalized- 


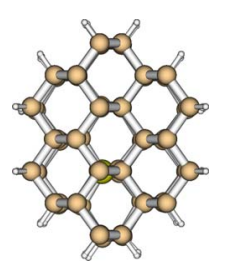

(a)
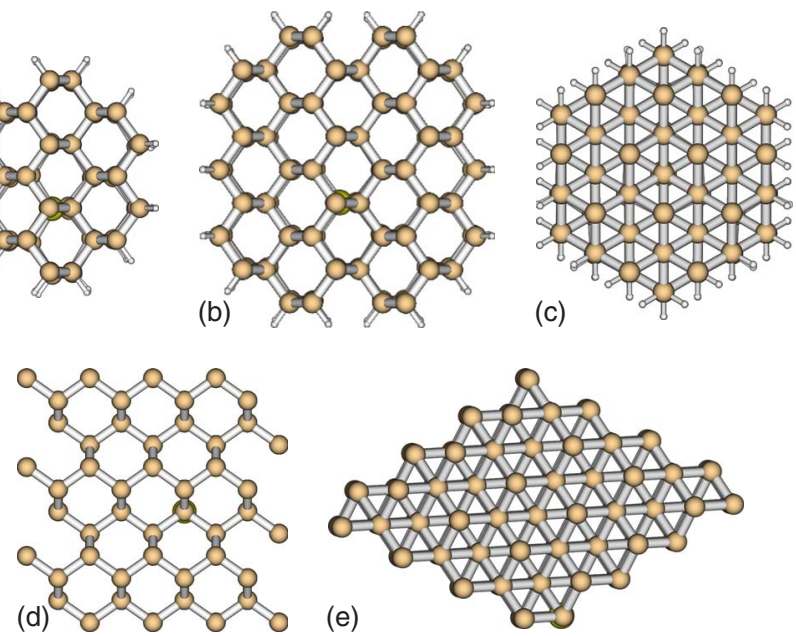

(e)

FIG. 1. (Color online) Cross sections of a $D=12 \AA\langle 110\rangle$ SiNW (a), a $D=17 \AA\langle 110\rangle$ SiNW (b), and a $D=16 \AA\langle 111\rangle$ SiNW (c). Bulk $4 \times 3$ (110) direction (d) and $4 \times 4$ (111) direction (e).

gradient approximation for the exchange-correlation functional. We use supercells containing either 7 unit cells (UCs) (for the $\langle 110\rangle$ wires) or 5 UCs (for the $\langle 111\rangle$ wires), with the metal impurity being positioned in the middlemost UC. The UC lengths are $L_{110}=3.9 \AA$ and $L_{111}=9.4 \AA$. Further, the structure is fully relaxed in the three middlemost UCs, see also Fig. 1(a)-1(c).

The DFT supercell is denoted as the central region and is described by the Kohn-Sham single-electron Hamiltonian matrix, $\mathbf{H}_{C}$, and overlap matrix $\mathbf{S}_{C}$. The zero-bias transmission function is calculated using nonequilibrium Green's functions as implemented in TRANSIESTA. ${ }^{25}$ Similar transmission calculations in SiNWs containing dopant impurities have previously been reported. ${ }^{22,26}$ In the transport calculations, the central region (the DFT supercell) is connected to two semi-infinite electrodes which are accounted for through a left $(\mathrm{L})$ and right $(\mathrm{R})$ self-energy, $\boldsymbol{\Sigma}_{L, R}(E)$. The transmission is calculated as

$$
T(E)=\operatorname{Tr}\left[\mathbf{G}_{C}^{r}(E) \boldsymbol{\Gamma}_{L}(E) \mathbf{G}_{C}^{a}(E) \boldsymbol{\Gamma}_{R}(E)\right],
$$

where $\mathbf{G}_{C}^{r}(E)=\left[E \mathbf{S}_{C}-\mathbf{H}_{C}-\mathbf{\Sigma}_{L}^{r}(E)-\boldsymbol{\Sigma}_{R}^{r}(E)\right]^{-1}$ is the retarded Green's function in the central region, and $\boldsymbol{\Gamma}_{\alpha}(E)=i$ $\left(\boldsymbol{\Sigma}_{\alpha}^{r}-\Sigma_{\alpha}^{a}\right)$. Due to time-reversal symmetry under steady state conditions, $\mathbf{G}_{C}^{a}=\left(\mathbf{G}_{C}^{r}\right)^{\dagger}$. The low-bias and low-temperature conductance of the wire is calculated by the Landauer formula, $G(E)=\left(2 e^{2} / h\right) T(E)$.

We assume that the impurities in a physical wire are distributed randomly along the wire. The transmission through a long wire with many impurities can be calculated recursively by successively adding small pieces of the wire. ${ }^{22,27} \mathrm{Calcu}-$ lations on many wires with different impurity configurations yield an ensemble averaged transmission, from where e.g., the elastic mean free path (MFP), $l_{e}$, can be extracted. Such calculations are rather time consuming because the sample averaging typically requires several hundred different configurations to reach converging results. However, following Refs. 22, 28, and 29, the MFP can be estimated from the single-impurity transmission, $T$, as

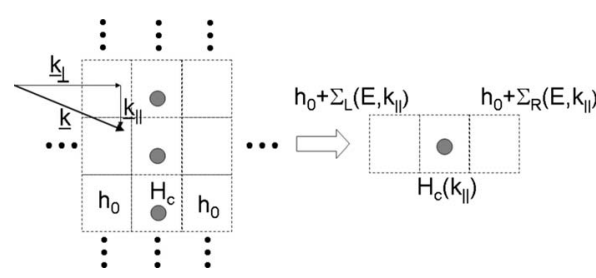

FIG. 2. Schematic 2D drawing of the bulk scattering method. $k_{\perp}$ denote the transport direction, and the transmission is $k$ point sampled over $k_{\|}$.

$$
l_{e}(E)=d \frac{T(E)}{T_{0}(E)-T(E)} .
$$

Here, $d$ is the average distance between impurities in the wire direction. $T_{0}(E)=N(E)$ is the pristine wire transmission with $N(E)$ being the number of conducting channels (bands) at energy $E$. We define a scattering cross section, $\sigma$, through the relation $\sigma=\left(l_{e} n\right)^{-1}$, where $n=(A d)^{-1}$ is the impurity density, and $A$ is the cross sectional area. It follows that the scattering cross section can be estimated from the singleimpurity transmission as

$$
\sigma(E)=A \frac{T_{0}(E)-T(E)}{T(E)} .
$$

The above considerations apply to any quasi-onedimensional structure such as nanowires or nanotubes. Due to computational limitations, transmission calculations are only feasible for ultrathin wires with diameters $D \leq 2 \mathrm{~nm}$, since otherwise the number of atoms becomes too large. However, most experimentally produced SiNWs are in the diameter range $10-100 \mathrm{~nm}$. To abridge the gap between $a b$ initio calculations and the typical experimental situation we have employed the following procedure. The thin wire results are compared to the scattering cross section in large wires, where the transport is dominated by the interior of the wire which is bulklike. Any finite-size quantization or surface effects will be ignored in this approach. The transmission equations to be calculated are in principle the same as above, but now we do not calculate the transport through a piece of wire, but rather through a piece of bulk $\mathrm{Si}$, that is periodically repeated in the direction transverse to the "wire"-direction, as indicated in Fig. 2. The transmission through the transverse unit cell is then calculated per area of the transverse cell using a dense $k$-point average and will correspond to the transmission though a certain plane [e.g., (111) or (110)] through the impurity. From the calculation with and without impurity we may again estimate the cross section via Eq. (3).

Consider now Au impurities in bulk $\mathrm{Si}$ in (111) and (110) directions with unit cell sizes of $4 \times 4$ and $4 \times 3$, respectively, corresponding to a Au-Au smallest distance of $15.5 \AA$, see Fig. 1(d) and 1(e). The electronic structure calculations and relaxations, similar to that of the wires, were performed for substitutional and tetragonal positions but now using a Monkhorst-Pack $k$-point sampling of $2 \times 2$ for (111) and 2 $\times 1$ for (110). The calculations without impurities were done using smaller cells $1 \times 1$ and $1 \times 2$ with corresponding 

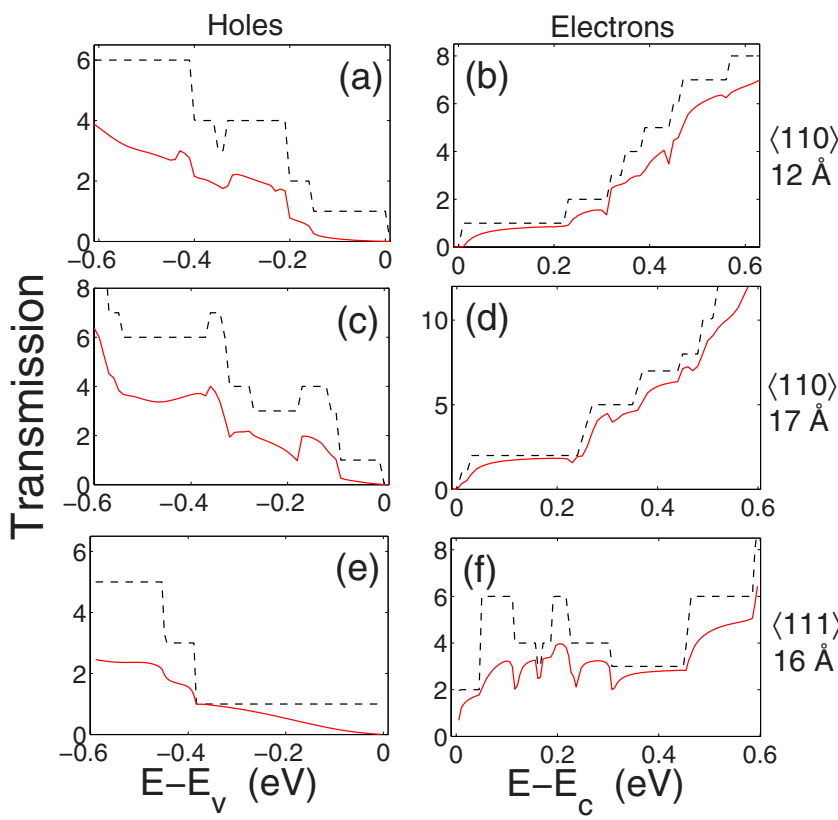

considered SiNWs with a single substitutional Au impurity (solid curves) together with the pristine wire transmissions (dashed curves). Left and right column correspond to holes and electrons with the energy axes being relative to the valence and conduction band edges, respectively. As a general trend we observe that the holes are scattered more strongly than the electrons. In particular close to the valence band edge there is a strong scattering with the transmission approaching zero. From Eq. (2) it follows that in this energy region the MFP $l_{e} \rightarrow 0$, while the scattering cross section $\sigma$ diverges. This is further illustrated in Fig. 4 showing $\sigma$ for the three SiNW calculations in Fig. 3 together with results obtained from bulk calculations.

In all wire and bulk calculations, $\sigma$ diverges close to the band edges but saturates further into the bands. We notice that the overall behavior appears to be independent of the wire orientation or size. Inside the valence band, $>0.2 \mathrm{eV}$ away from the band edge, $\sigma$ lies in the range 100-200 $\AA^{2}$, while in the conduction band, $\sigma$ is in the range 10-100 $\AA^{2}$.

The oscillations in the $\sigma$ curves for the wire calculations reflect the band structures of the SiNWs. The bulk valence and conduction bands are folded into the wire direction giving rise to many valence and conduction bands. This is also seen in the transmission plots, Fig. 3, where each step in the pristine wire transmissions reflects a band edge. At the beginning of a new band, the pristine wire transmission, $T_{0}(E)$, abruptly increases while the transmission through the SiNWs with impurities increases more slowly. Consequently $\sigma$ increases abruptly at the subband edges, seen as the peaks in the SiNW results. The bulk calculated $\sigma$ shows, on the other hand, a smoothly decaying behavior into both the valence and conduction bands.

It is encouraging to see that there is an overall consistency between the wire results and the bulk calculations. Both methods predict that $\sigma$ is larger in the valence band than in the conduction band, and the divergence close to the band edge is also captured by both methods. This suggests that it is possible to define a meaningful quantity, $\sigma$, which characterizes the scattering properties of Au impurities in Si. This is potentially an important finding, since now, e.g., the MFP of much larger wires can be estimated as $l_{e}(E)=n / \sigma(E)$, without the need to perform very time consuming calculations on large structures.

\section{B. Comparison of Au positions}

\section{A. Au substitutional impurity}

In Fig. 3 we plot the transmission functions for the three
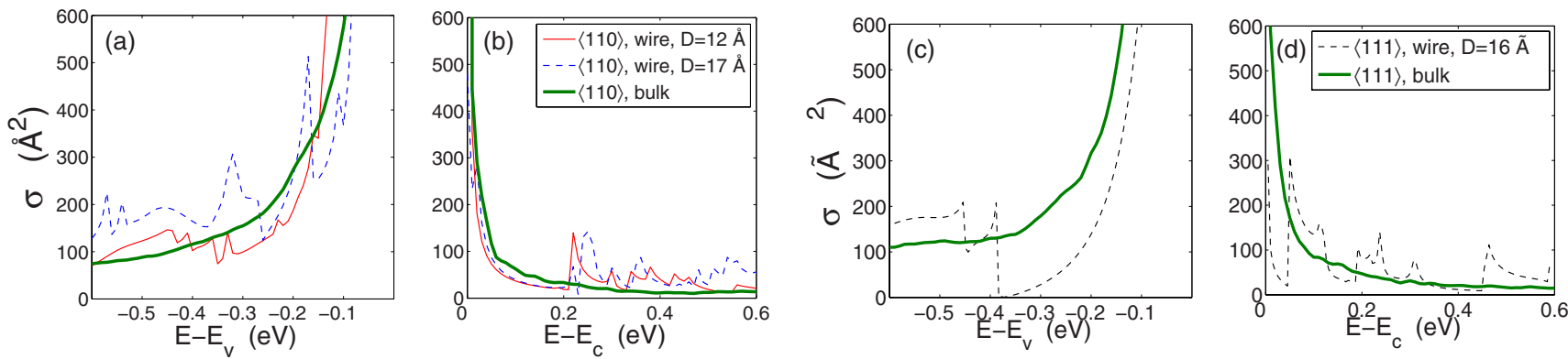

FIG. 4. (Color online) Scattering cross sections of $\langle 110\rangle$ wires (a) and (b) and of the $\langle 111\rangle$ wire (c) and (d). Bulk results are show with thick lines. The SiNW results are obtained from the transmission shown in Fig. 3. 

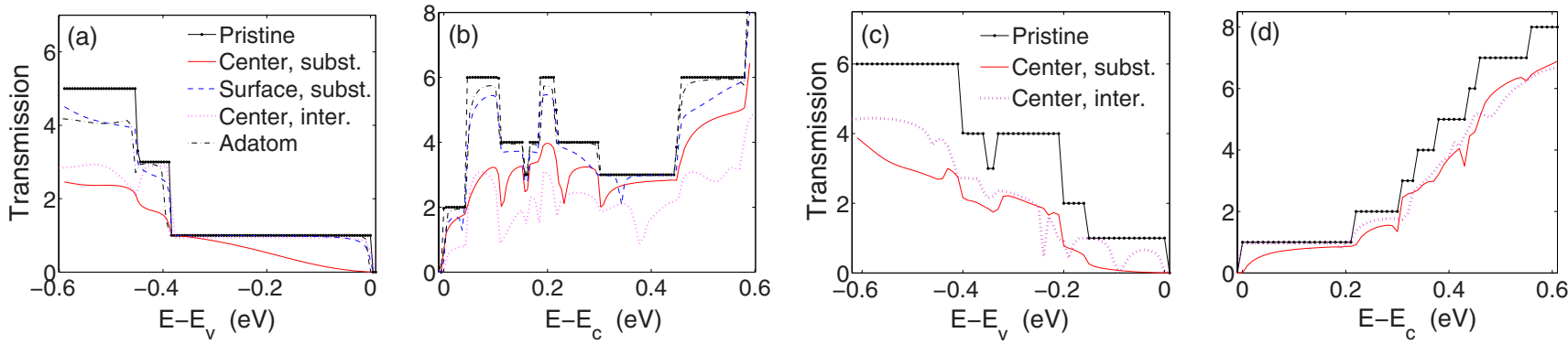

FIG. 5. (Color online) Transmission through $D=16 \AA\langle 111\rangle$ SiNWs (a)-(b) with the Au atom in different positions: Substitutional in the center of the wire (solid), substitutional at the surface (dashed), interstitial in the center (small dotted), surface adatom (dash dotted). The pristine wire transmission is indicated by larger dots. Panels (c) and (d) show transmission through the $D=12 \AA\langle 110\rangle$ SiNW with the Au in a substitutional and interstitial position in the center of the wire.

favorable configuration, ${ }^{19}$ other positions are possible. Two different interstitial positions, a tetrahedral and a hexagonal, were observed in experiments. ${ }^{19}$ We have performed calculations on both interstitial positions, but upon structural relaxation, the hexagonal position relaxed to the tetrahedral position. This is probably due to finite-size effects in the SiNWs considered. Because of their reduced diameter, the only hexagonal interstitial position available is very close to the wire surface which might make it unstable vs relaxation toward a tetrahedral interstitial site.

The substitutional position might be at different radial locations, with correspondingly different scattering properties. Studies of phosphorous (P) impurities in SiNWs have indeed shown that the radial position of the $\mathrm{P}$ atoms has a strong influence on the impurity limited mean free path. ${ }^{29}$ Yet another possible impurity site is at the SiNW surface as an adatom.

Figure 5 shows the transmission through SiNWs with the $\mathrm{Au}$ impurity being in different positions. Panels (a) and (b) correspond to energies relative to the valence and conduction band edges, respectively, for the $D=16 \AA\langle 111\rangle$ wire. The substitutional position in the center of the wire (solid line) is clearly the strongest scatterer in the valence band, where all other positions show a high degree of transparency in the first transmission plateau. In the conduction band, the central substitutional position is still rather strongly scattering, but the tetrahedral interstitial position (dotted line) scatters the electrons even more. The surface substitutional as well as the adatom positions are very weak scatterers also in the conduction band. The formation energies ${ }^{31}$ of the considered configurations yield a surface segregation energy of $0.8 \mathrm{eV}$, similarly to previous reports on doping impurities in both SiNWs (Refs. 32 and 33 ) and Ge nanowires ${ }^{34}$ and in agreement with experimental results. ${ }^{35,36}$

Panels (c) and (d) in Fig. 5 show results for the $D$ $=12 \AA\langle 110\rangle$ SiNW with the Au in a substitutional and interstitial position in the center of the wire. As for the $\langle 111\rangle$ wire, the interstitial positions scatter the holes less than the substitutional position. An exception is the transmission dip around $E-E_{v}=-0.1 \mathrm{eV}$ where a quasilocalized state gives rise to Fano antiresonances ${ }^{37}$ and complete back scattering. Such resonant back scattering has previously been discussed in the context of SiNWs for both neutral ${ }^{26}$ and charged impurities. ${ }^{38}$ Contrary to the $\langle 111\rangle$ SiNW, the interstitial Au position is a much weaker scatterer close to the conduction band edge, with an almost perfect transmission.

The trends found for the substitutional and interstitial positions in the center of the wires are qualitatively reproduced in the bulk calculations. Figure 6 shows the scattering cross section for $\langle 110\rangle$ and $\langle 111\rangle$ directions with the Au being in either a substitutional or tetrahedral interstitial position. The bulk calculations show two main trends (i): In the valence band (a), the interstitial position causes less scattering (i.e., has a smaller $\sigma$ ) than the substitutional position. (ii) In the conduction band (b), the interstitial position has the smallest $\sigma$ for transport in the $\langle 110\rangle$ direction but has the largest $\sigma$ for transport in the $\langle 111\rangle$ direction. Similar results are found for the SiNW calculations as shown in Fig. 5, providing further evidence that the bulk and the wire calculations are consistent with each other.

\section{Comparison of different metals}

$\mathrm{Au}$ is by far the most common catalyst in the VLS growth but, as already mentioned, also other metals have been used. In this section we compare the scattering cross sections of $\mathrm{Au}, \mathrm{Ag}, \mathrm{Al}$, and $\mathrm{Cu}$ metal impurities. We consider only the substitutional position in the center of the wire. Figure 7 shows the calculated $\sigma$ for the same three SiNWs studied above. Notice the different scales in the subfigures. Evidently, the group 11 metals $\mathrm{Cu}, \mathrm{Ag}$, and $\mathrm{Au}$ all behave very
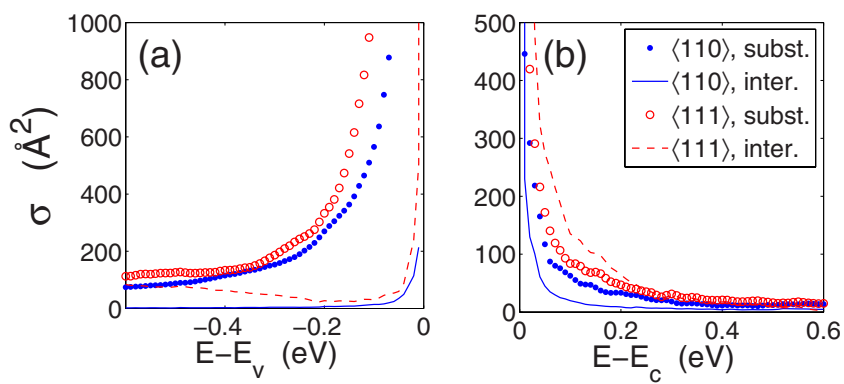

FIG. 6. (Color online) Scattering cross section calculated with the bulk method. Filled and empty circles correspond to a substitutional $\mathrm{Au}$ position with transport in the $\langle 110\rangle$ and $\langle 111\rangle$ directions, respectively. Likewise, solid and dashed lines correspond to an interstitial $\mathrm{Au}$ position for transport in the $\langle 110\rangle$ and $\langle 111\rangle$ directions, respectively. 

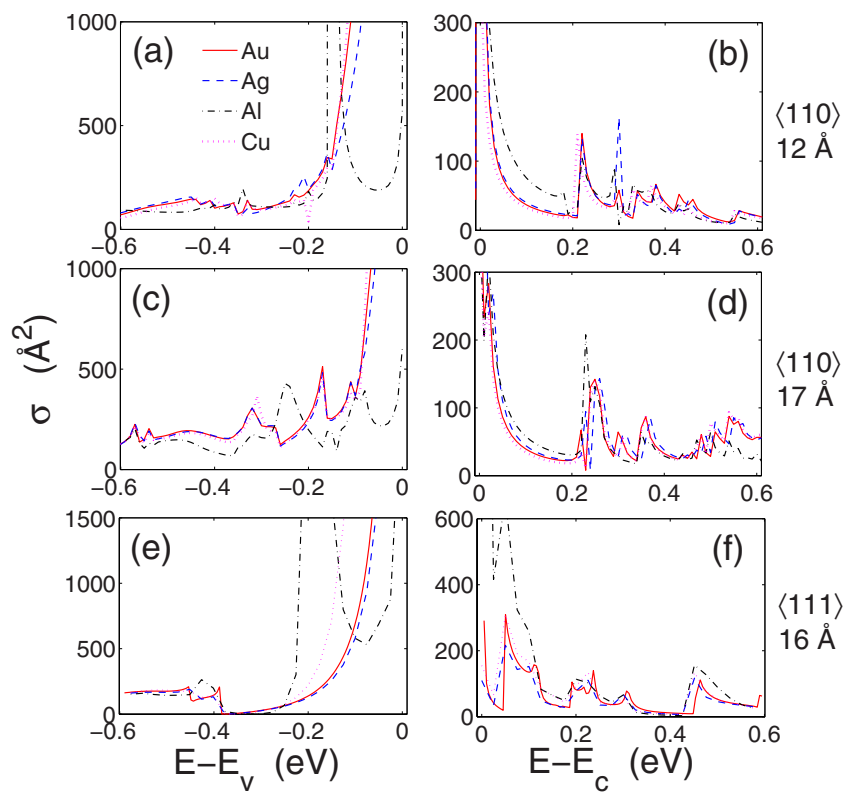

FIG. 7. (Color online) Scattering cross section, $\sigma$, for substitutional positions in the center of the wires. (a) and (b) correspond to the $D=12 \AA\langle 110\rangle$ wire, (c) and (d) to the $D=17 \AA\langle 110\rangle$ wire, (e) and (f) to the $D=16 \AA\langle 111\rangle$ wire.

similarly. The average nearest neighbor bond lengths in the relaxed geometries are $d_{\mathrm{Au}-\mathrm{Si}}=2.42 \AA, d_{\mathrm{Ag}-\mathrm{Si}}=2.46 \AA$, $d_{\mathrm{Cu}-\mathrm{Si}}=2.34 \AA$, and $d_{\mathrm{Al}-\mathrm{Si}}=2.48 \AA$, while the average $\mathrm{Si}-\mathrm{Si}$ nearest neighbor distance is $2.42 \AA$. The similar behavior of $\mathrm{Cu}, \mathrm{Ag}$, and $\mathrm{Au}$ and the differences from $\mathrm{Al}$ cannot be explained from the relaxed geometries, and the transport characteristics seem to be completely dominated by the valence of the impurities.

$\mathrm{Al}$ differs from the other metals in particular at energies in the valence band. Close to the valence band edge, Al has a significantly smaller scattering cross section than the others. In the $D=12 \AA\langle 110\rangle$ and $D=16 \AA\langle 111\rangle$ wires a clear peak in $\sigma$ for $\mathrm{Al}$ is observed around $E-E_{V}=-0.2 \mathrm{eV}$. The strong $\mathrm{Al}$ scattering in this energy range is due to quasi localized states giving rise to resonant back scattering.

Based on the calculated scattering cross sections, there is no clear preference for one of the metals to use as catalyst. While the group 11 metals practically have identical $\sigma, \mathrm{Al}$ has a smaller $\sigma$ close to the valence band edge, but a larger $\sigma$ close to the conduction band edge. It therefore does not seem necessary to consider the scattering properties when choosing the metal catalyst.

\section{DISCUSSION}

At face value, it may seem unphysical that an impurity in a SiNW with a finite cross sectional area, $A$, can have a diverging scattering cross section, as is the case close to the band edges. One might argue that a proper definition of $\sigma$ should lead to a maximum value equal to the wire area, $A$. It is possible to define an alternative scattering cross section, $\sigma^{\prime}(E)$, with this property through the relation
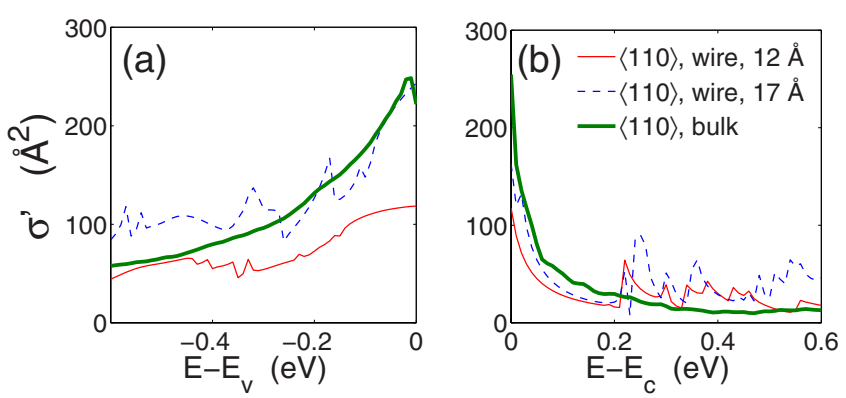

FIG. 8. (Color online) Scattering cross section, $\sigma^{\prime}$, given by Eq. (5) for the same data as shown in Figs. 4(a) and 4(b).

$$
T(E)=\left[A-\sigma^{\prime}(E)\right] \tau(E),
$$

where $\tau(E)$ is defined by the pristine wire transmission $T_{0}(E)=A \tau(E)$, with $A$ being the cross sectional area of the wire. It follows that $\sigma^{\prime}(E)$ can be written as

$$
\sigma^{\prime}(E)=A \frac{T_{0}(E)-T(E)}{T_{0}(E)}=\frac{A \sigma(E)}{A+\sigma(E)} .
$$

In the limit $\sigma \rightarrow \infty$ the new scattering cross section is finite, $\sigma^{\prime} \rightarrow A$. Conversely, in the weak scattering limit, $\sigma \ll A$, the two definitions of the scattering cross section coincide. The interpretation of $\sigma^{\prime}$ as defined in Eq. (4) is that an impurity effectively blocks an area of the wire, where the electrons no longer can transmit through. A strong scatterer occupies a large area while a weak scatterer occupies a small area. While the definition of $\sigma^{\prime}$ is rather intuitive, the results obtained with this definition are not straightforwardly interpreted. Figure 8 shows the data from Figs. 4(a) and 4(b) transformed to $\sigma^{\prime}$. Instead of diverging close to the band edges, $\sigma^{\prime}$ is bounded from above by the different cross sectional areas in the calculations. The different $\sigma^{\prime}$ curves differ from each other more than the corresponding $\sigma$ curves in Fig. 4 , in particular close to the band edges. Consequently, $\sigma$ seems to be a better descriptor of the scattering properties of an impurity, independently on the wire size.

The diverging $\sigma$ close to the band edges results from the MFP going to zero. This result may readily be obtained from a simple model. Consider a single band model with parabolic band structure $\varepsilon(k)=\frac{\hbar^{2} k^{2}}{2 m^{*}}$ and effective mass $m^{*}$. The MFP, $l_{e}(E)$, can be written as

$$
l_{e}(E)=v(E) \tau(E),
$$

where $v(E)=\sqrt{2 E / m^{*}}$ and $\tau(E)$ are the group velocity and transport relaxation time at energy $E$. The latter can be estimated from Fermi's golden rule (FGR) as

$$
\frac{1}{\tau(E)}=\frac{2 \pi}{\hbar} \int \frac{d k^{\prime}}{2 \pi}\left|M_{k^{\prime}, k}\right|^{2}\left(1-\cos \theta_{k, k^{\prime}}\right) \delta\left[\varepsilon\left(k^{\prime}\right)-\varepsilon(k)\right],
$$

where $\theta_{k, k^{\prime}}$ is the angle between the initial and final wave vectors. We will only consider scattering from a forward moving initial state to a backward moving final state. Therefore, $\theta_{k, k^{\prime}}=-\pi$, yielding a constant factor of 2 . To simplify the discussion, we assume that the matrix element $\left|M_{k^{\prime}, k}\right|^{2}$ 
$=\left|\left\langle k^{\prime}\left|V_{\text {imp }}(\mathbf{r})\right| k\right\rangle\right|^{2}$ is a constant, $|M|^{2}$ times the impurity concentration, $n$. Here, $V_{i m p}(\mathbf{r})$ is the impurity potential from a single impurity, $|k\rangle$ is a Bloch state with wave number $k$, and $\varepsilon(k)=E$. The relaxation time can now be written as

$$
\frac{1}{\tau(E)}=\frac{4 \pi}{\hbar} n|M|^{2} D(E),
$$

where $D(E)=(\pi \hbar)^{-1} \sqrt{m^{*} / E}$ is the density of state. It follows that the scattering cross section becomes

$$
\sigma(E)=\frac{1}{n l_{e}(E)}=\frac{2 \sqrt{2} m^{*}|M|^{2}}{\hbar^{2} E} .
$$

Within this simple model the scattering cross section is proportional to the effective mass and inversely proportional to the energy, and thus diverges close to the band edge, in agreement with the numerical results presented above.

\section{SUMMARY AND CONCLUSION}

We have considered the scattering properties of metal catalyst atoms in SiNWs and bulk Si. The analysis is based on first-principles density functional theory combined with nonequilibrium Green's functions.

Among different metal catalysts we have found that the group 11 metals $\mathrm{Au}, \mathrm{Ag}$, and $\mathrm{Cu}$ have very similar scattering properties, while $\mathrm{Al}$ differs from the others particularly for hole transport in the valence band. Based on our analysis the scattering properties of different metal atoms does not appear as an important design parameter, as the different metals scatters the electrons similarly.

By defining a scattering cross section, $\sigma(E)$, we have shown that the energy dependent scattering strength of a given impurity can be consistently calculated in SiNWs as well as in bulk $\mathrm{Si} . \sigma(E)$ gives a fingerprint for a specific impurity in a given transport direction which can be obtained through different calculations. The good agreement between trends found in the SiNW and bulk calculations show that finite size and surface effects are not dominating the scattering properties, even though the considered SiNWs have diameters below $2 \mathrm{~nm}$. This indicates that scattering properties of defects obtained for ultrathin wires can be generalized to larger and experimentally more relevant wires.

\section{ACKNOWLEDGMENTS}

We thank the Danish Center for Scientific Computing (DCSC) and Direktør Henriksens Fond for providing computer resources. A.P.J. is grateful to the FiDiPro program of the Finnish Academy. Financial support by the Ramón y Cajal program of the Ministerio de Ciencia e Innovación and funding under Contracts No. TEC2006-13731-C02-01, No. TEC2009-06986, and No. FIS2009-12721-C04-03 are greatly acknowledged.
*Present address: Center for Atomic-scale Materials Design (CAMD), Department of Physics, Technical University of Denmark, DK-2800 Kgs. Lyngby, Denmark.

${ }^{1}$ Y. Cui and C. M. Lieber, Science 291, 851 (2001).

${ }^{2}$ M. S. Gudiksen, L. J. Lauhon, J. Wang, D. C. Smith, and C. M. Lieber, Nature (London) 415, 617 (2002).

${ }^{3}$ Y. Cui, Z. Zhong, D. Wang, W. U. Wang, and C. M. Lieber, Nano Lett. 3, 149 (2003).

${ }^{4}$ Y. Wu, C. Yang, W. Lu, and C. M. Lieber, Nature (London) 430 , 61 (2004).

${ }^{5}$ L. Samuelson, Mater. Today 6, 22 (2003).

${ }^{6}$ F. Patolsky and C. M. Lieber, Mater. Today 8, 20 (2005).

${ }^{7}$ A. Hochbaum, R. Chen, R. D. Delgado, W. Liang, E. C. Garnett, M. Najarian, A. Marumdar, and P. Yang, Nature (London) 451, 163 (2008).

${ }^{8}$ A. I. Boukai, Y. Bunimovich, J. Tahir-Kheli, J.-K. Yu, W. A. Goddard III, and J. R. Heath, Nature (London) 451, 168 (2008).

${ }^{9}$ R. Rurali, Rev. Mod. Phys. 82, 427 (2010).

${ }^{10}$ R. S. Wagner and W. C. Ellis, Appl. Phys. Lett. 4, 89 (1964).

${ }^{11}$ Y. Cui, L. J. Lauhon, M. S. Gudiksen, J. Wang, and C. M. Lieber, Appl. Phys. Lett. 78, 2214 (2001).

${ }^{12}$ Y. Wu, Y. Cui, L. Huynh, C. Barrelet, D. Bell, and C. Lieber, Nano Lett. 4, 433 (2004).

${ }^{13}$ J. D. Holmes, K. P. Johnston, R. C. Doty, and B. A. Korgel, Science 287, 1471 (2000)

${ }^{14}$ H. Fang, Y. Wu, J. Zhao, and J. Zhu, Nanotechnology 17, 3768 (2006).

${ }^{15}$ Y. Wang, V. Schmidt, S. Senz, and U. Gösele, Nat. Nanotechnol.
1, 186 (2006).

${ }^{16}$ Y. Yao and S. Fan, Mater. Lett. 61, 177 (2007).

${ }^{17}$ J. Arbiol and B. Kalache, P. R. i Cabarrocas, J. R. Morante, and A. F. i Morral, Nanotechnology 18, 305606 (2007).

${ }^{18}$ T. Baron, M. Gordon, F. Dhalluin, C. Ternon, P. Ferret, and P. Gentile, Appl. Phys. Lett. 89, 233111 (2006).

${ }^{19}$ S. H. Oh, K. v. Benthem, S. I. Molina, A. Y. Borisevich, W. Luo, P. Werner, N. D. Zakharov, D. Kumar, S. T. Pantelides, and S. J. Pennycook, Nano Lett. 8, 1016 (2008).

${ }^{20}$ D. E. Perea, J. E. Allen, S. J. May, B. W. Wessels, D. N. Seidman, and L. J. Lauhon, Nano Lett. 6, 181 (2006).

${ }^{21}$ C. B. Collins, R. O. Carlson, and C. J. Gallagher, Phys. Rev. 105, 1168 (1957).

${ }^{22}$ T. Markussen, R. Rurali, A.-P. Jauho, and M. Brandbyge, Phys. Rev. Lett. 99, 076803 (2007).

${ }^{23}$ J. M. Soler, E. Artacho, J. D. Gale, A. García, J. Junquera, P. Ordejón, and D. Sánchez-Portal, J. Phys.: Condens. Matter 14, 2745 (2002).

${ }^{24}$ E. Anglada, J. M. Soler, J. Junquera, and E. Artacho, Phys. Rev. B 66, 205101 (2002).

${ }^{25}$ M. Brandbyge, J.-L. Mozos, P. Ordejón, J. Taylor, and K. Stokbro, Phys. Rev. B 65, 165401 (2002).

${ }^{26}$ M.-V. Fernández-Serra, Ch. Adessi, and X. Blase, Nano Lett. 6, 2674 (2006).

${ }^{27}$ T. Markussen, R. Rurali, M. Brandbyge, and A.-P. Jauho, Phys. Rev. B 74, 245313 (2006).

${ }^{28}$ T. Markussen, A.-P. Jauho, and M. Brandbyge, Phys. Rev. B 79, 035415 (2009). 
${ }^{29}$ T. Markussen, R. Rurali, A.-P. Jauho, and M. Brandbyge, J. Comput. Electron. 7, 324 (2008).

${ }^{30}$ Since $T_{0}$ and $T$ come from two separate calculations we find that the exact values for the band edges can be shifted slightly between these. This may change the bulk scattering cross sections significantly for energies close to the band edge where $T_{0}(E)$ goes abruptly to zero. We therefore introduce a small energy shift, $\delta$, determined such that $T(E+\delta) / T_{0}(E) \approx 0$ for $E_{b}$ close to the band edges $E_{C}, E_{V}$ as obtained from the $T_{0}$ calculation.

${ }^{31}$ R. Rurali and X. Cartoixà, Nano Lett. 9, 975 (2009).

${ }^{32}$ M. V. Fernández-Serra, Ch. Adessi, and X. Blase, Phys. Rev. Lett. 96, 166805 (2006).
${ }^{33}$ H. Peelaers, B. Partoens, and F. Peeters, Nano Lett. 6, 2781 (2006).

${ }^{34}$ H. Peelaers, B. Partoens, and F. M. Peeters, Appl. Phys. Lett. 90, 263103 (2007).

${ }^{35}$ J. B. Hannon, S. Kodambaka, F. M. Ross, and R. M. Tromp, Nature (London) 440, 69 (2006).

${ }^{36}$ J. E. Allen, E. R. Hemesath, D. E. Perea, J. L. Lensch-Falk, Z. Y. Li, F. Yin, M. H. Gass, P. Wang, A. L. Bleloch, R. E. Palmer, and L. J. Lauhon, Nat. Nanotechnol. 3, 168 (2008).

${ }^{37}$ J. U. Nockel and A. D. Stone, Phys. Rev. B 50, 17415 (1994).

${ }^{38}$ R. Rurali, T. Markussen, J. Sũné, M. Brandbyge, and A.-P. Jauho, Nano Lett. 8, 2825 (2008). 